\title{
Texture simulation based on tensorial Fourier coefficients
}

\author{
Thomas Böhlke \\ Otto-von-Guericke-Universität, University of Magdeburg, Institute of Mechanics, Postfach 4120, D-39016 Magdeburg, Germany
}

Received 15 August 2005; accepted 18 January 2006

\begin{abstract}
The main result of the paper is the derivation of the evolution equation of the tensorial texture coefficients of the crystallite orientation distribution function (codf). The evolution equation of each coefficient depends on the complete codf and the lattice spin, which is a constitutive quantity. For the solution of the differential equation based on a finite number of coefficients, the codf has to be estimated. This estimate is obtained here by the maximum entropy method. By this approach the texture evolution can be described by modeling some low-order Fourier coefficients. It will be shown that such a low-dimensional approach yields a reasonable description of the texture evolution and of mechanical properties like the Taylor factor.
\end{abstract}

(C) 2006 Civil-Comp Ltd. and Elsevier Ltd. All rights reserved.

PACS: $83.50 . \mathrm{La}$

Keywords: Anisotropic viscoplasticity; Crystallite orientation distribution function; Crystallographic texture; Evolution equation of texture coefficients; Maximum entropy method; Tensorial Fourier expansion

\section{Introduction}

Phenomenological models seem to be generally unable to adequately represent the evolution of the crystallite orientation distribution. Therefore, if the evolving crystallographic texture has to be taken into account in the context of finite element simulations in most cases the Taylor model is applied $[18,14,19]$. In this case, the texture evolution is described by a system of ordinary or algebro differential equations. The dimension of such a system is in between 1000 and 10000 . Hence, at each integration point of the finite element mesh large systems of differential equations have to be integrated. This fact considerably limits the number of degrees of freedom that can be handled by standard finite element codes. Therefore, there is a need for homogenization strategies which allow to condense the number of degrees of freedom and nevertheless accurately describe the crystallite orientation distribution function (codf).

E-mail address: boehlke@mb.uni-magdeburg.de
Here an approach is presented that is based on a tensorial Fourier expansion of the codf $[1,10]$. The tensorial Fourier coefficients or texture coefficients can be considered as micro-mechanically based tensorial internal variables $[5$, $6,22]$. They are defined in terms of the codf, which can be determined by texture measurements. The main result of the paper is the derivation of the evolution equation of the tensorial texture coefficients. The evolution equation of each coefficient depends on the complete codf and on the lattice spin, which is a constitutive quantity. Hence, for a solution of the differential equation based on a finite number of coefficients, the codf has to be estimated. This estimate is obtained here by the maximum entropy method [4]. Based on the aforementioned approach the texture evolution can be described by modeling some low-order Fourier coefficients. It will be shown that such a low-dimensional approach yields a reasonable description of the texture evolution.

The outline of the paper is given as follows. Section 2 gives a summary concerning the representation of the codf by a tensorial Fourier expansion. In Section 3 the evolution 
equation of the texture coefficients is derived for the special case of a homogeneous deformation of the aggregate. In Section 4 a numerical example is considered. The texture evolution is predicted for a simple shear deformation based on both a representation of the codf by a discrete orientation distribution and a continuous representation based on the leading texture coefficients. It is shown that the second approach allows to reduce the number of degrees of freedom significantly.

Notation. Fourth- and higher-order tensors are denoted by, e.g., $\mathbb{V}_{\langle\alpha\rangle}$, where the index $\alpha$ specifies the tensor rank. The corresponding zero tensor is written as $\mathbb{O}$. The dyadic product and the Frobenius norm are denoted by $\otimes$ and $\|\cdot\|$, respectively. Components of tensors are always represented with respect to an orthonormal vector basis $\boldsymbol{e}_{i}$ in the threedimensional Euclidean space. For example, a fourth-order tensor is written in the following way: $\mathbb{V}_{\langle 4\rangle}=V_{i j k l} \boldsymbol{e}_{i} \otimes \boldsymbol{e}_{j} \otimes$ $\boldsymbol{e}_{k} \otimes \boldsymbol{e}_{l}$. The scalar product between tensors is denoted by a dot, e.g., $\mathbb{V}_{\langle\alpha\rangle} \cdot \mathbb{F}_{\langle\alpha\rangle}$. A linear mapping is written as $\mathbb{V}_{\langle\alpha\rangle}=$ $\mathbb{E}_{\langle 2 \alpha\rangle}\left[\mathbb{F}_{\langle\alpha\rangle}\right]$. Irreducible, i.e., completely symmetric and traceless, tensors are designated by a prime, e.g., $\mathbb{V}_{\langle\alpha\rangle}^{\prime}$. The number of independent components of a tensor $\mathbb{V}_{\langle\alpha\rangle}$ is denoted by $\operatorname{dim}\left(\mathbb{V}_{\langle\alpha\rangle}\right)$. If it is necessary to distinguish between tensors of the same rank, which are denoted by the same capital letter, then an additional index in the bracket specifying the tensor rank is introduced. For example, $\mathbb{V}_{\left\langle 4_{1}\right\rangle}^{\prime}$ and $\mathbb{V}_{\left\langle 4_{2}\right\rangle}^{\prime}$ are two different fourth-order tensors. The set of proper orthogonal tensors is specified by $S O(3)$. A superimposed dot denotes the material time derivative.

\section{Tensorial representation of the codf}

\subsection{Properties of the codf}

A crystal orientation is described by a proper orthogonal tensor $\boldsymbol{Q}=\boldsymbol{g}_{i} \otimes \boldsymbol{e}_{i} \in S O(3)$ which is introduced in such a way that it maps the fixed reference basis $\boldsymbol{e}_{i}$ onto the lattice vectors $\boldsymbol{g}_{i}$. $\boldsymbol{Q}$ can be parameterized by Euler angles $\phi_{1}=\varphi_{1} \in[0,2 \pi), \phi_{2}=\Phi \in[0, \pi], \phi_{3}=\varphi_{2} \in[0,2 \pi)[8]$

$$
\left(\boldsymbol{Q}^{\top}\right)_{i j}=\left[\begin{array}{ccc}
C_{1} C_{3}-S_{1} C_{2} S_{3} & S_{1} C_{3}+C_{1} C_{2} S_{3} & S_{2} S_{3} \\
-C_{1} S_{3}-S_{1} C_{2} C_{3} & -S_{1} S_{3}+C_{1} C_{2} C_{3} & S_{2} C_{3} \\
S_{1} S_{2} & -C_{1} S_{2} & C_{2}
\end{array}\right],
$$

where $C_{i}$ and $S_{i}$ denote the values $\cos \left(\phi_{i}\right)$ and $\sin \left(\phi_{i}\right)$, respectively. The matrix components refer to the base vectors $\boldsymbol{e}_{i}$. The transposition is introduced in order to make the description of crystal orientations by $\boldsymbol{Q}=\boldsymbol{g}_{i} \otimes \boldsymbol{e}_{i}$ compatible to the one introduced by Bunge [8].

The codf $f(\boldsymbol{Q})$ specifies the volume fraction $\mathrm{d} v / v$ of crystals having the orientation $\boldsymbol{Q}[7,20]$, i.e.,

$\frac{\mathrm{d} v}{v}(\boldsymbol{Q})=f(\boldsymbol{Q}) \mathrm{d} Q$.

$\mathrm{d} Q$ is the volume element in $S O(3)$ which ensures an invariant integration over $S O(3)$ [9], i.e.,
$\int_{S O(3)} f(\boldsymbol{Q}) \mathrm{d} Q=\int_{S O(3)} f\left(\boldsymbol{Q} \boldsymbol{Q}_{0}\right) \mathrm{d} Q \quad \forall \boldsymbol{Q}_{0} \in S O(3)$.

If $S O(3)$ is parameterized by Euler angles, the volume element $\mathrm{d} Q$ is given by

$\mathrm{d} Q=\frac{\sin (\Phi)}{8 \pi^{2}} \mathrm{~d} \varphi_{1} \mathrm{~d} \Phi \mathrm{d} \varphi_{2}$.

The function $f(\boldsymbol{Q})$ is nonnegative and normalized such that

$f(\boldsymbol{Q}) \geqslant 0 \quad \forall \boldsymbol{Q} \in S O(3), \quad \int_{S O(3)} f(\boldsymbol{Q}) \mathrm{d} Q=1$.

The orientation distribution function $f(\boldsymbol{Q})$ reflects both the symmetry of the crystallites forming the aggregate and the sample symmetry, which results from the processing history Zheng and $\mathrm{Fu}$ [25]. The crystal symmetry implies the following symmetry relation for $f(\boldsymbol{Q})$

$f(\boldsymbol{Q})=f\left(\boldsymbol{Q} \boldsymbol{H}^{\mathrm{C}}\right) \quad \forall \boldsymbol{H}^{\mathrm{C}} \in S^{\mathrm{C}} \subseteq S O(3)$.

$S^{\mathrm{C}}$ denotes the symmetry group of the crystallite. The sample symmetry implies the following symmetry relation for $f(\boldsymbol{Q})$

$f(\boldsymbol{Q})=f\left(\boldsymbol{H}^{\mathrm{S}} \boldsymbol{Q}\right) \quad \forall \boldsymbol{H}^{\mathrm{S}} \in S^{\mathrm{S}} \subseteq S O(3)$.

$S^{\mathrm{S}}$ denotes the symmetry group of the sample.

\subsection{Tensorial Fourier expansion of the codf}

For the subsequent considerations it is assumed that the codf is square integrable. This property implies the existence of a tensorial Fourier expansion. Adams et al. [1] and Guidi et al. [10] considered this expansion for the special case of a cubic crystal symmetry. Zheng and $\mathrm{Fu}[24,25]$ analyzed the expansion for arbitrary crystal and sample symmetries.

For aggregates of cubic crystals the Fourier expansion has the following form

$f(\boldsymbol{Q})=1+\sum_{i=1}^{\infty} f_{\alpha_{i}}(\boldsymbol{Q})$,

where

$f_{\alpha_{i}}=\mathbb{V}_{\left\langle\alpha_{i}\right\rangle}^{\prime} \cdot \mathbb{F}_{\left\langle\alpha_{i}\right\rangle}^{\prime}(\boldsymbol{Q}), \quad \mathbb{F}_{\left\langle\alpha_{i}\right\rangle}^{\prime}(\boldsymbol{Q})=\boldsymbol{Q} * \mathbb{T}_{\left\langle\alpha_{i}\right\rangle}^{\prime}$

and

$\left\{\alpha_{i}\right\}=\left\{4,6,8,9,10,12_{1}, 12_{2}, 13,14, \ldots\right\}$.

The $\mathbb{V}_{\left\langle\alpha_{i}\right\rangle}^{\prime}$ are called tensorial Fourier coefficients or texture coefficients. The tensors $\mathbb{T}_{\left\langle\alpha_{i}\right\rangle}^{\prime}$ are called reference tensors which are normalized without loss of generality

$\left\|\mathbb{T}_{\left\langle\alpha_{i}\right\rangle}^{\prime}\right\|=1$.

The * in Eq. $(9)_{2}$ denotes the Rayleigh product, which for tensors $\mathbb{T}=T_{i j \ldots l} \boldsymbol{e}_{i} \otimes \boldsymbol{e}_{j} \otimes \cdots \otimes \boldsymbol{e}_{l}$ of arbitrary rank is defined by

$\boldsymbol{Q} * \mathbb{T}=T_{i j \ldots l}\left(\boldsymbol{Q} \boldsymbol{e}_{i}\right) \otimes\left(\boldsymbol{Q} \boldsymbol{e}_{j}\right) \otimes \cdots \otimes\left(\boldsymbol{Q} \boldsymbol{e}_{l}\right)$.

The product $\boldsymbol{Q} * \mathbb{T}_{\left\langle\alpha_{i}\right\rangle}^{\prime}$ can be interpreted as the rotation of the tensor $\mathbb{T}_{\left\langle\alpha_{i}\right\rangle}^{\prime}$ by the orthogonal tensor $\boldsymbol{Q}$. The $\mathbb{V}_{\left\langle\alpha_{i}\right\rangle}^{\prime}$ and 
$\mathbb{T}_{\left\langle\alpha_{i}\right\rangle}^{\prime}$ are completely symmetric and traceless tensors. For example, the following relations hold for $\mathbb{V}_{\langle 4\rangle}^{\prime}$

$V_{i j k l}^{\prime}=V_{j i k l}^{\prime}=V_{k l i j}^{\prime}=V_{k j l}^{\prime}=\cdots, \quad V_{i i k l}^{\prime}=0$.

Completely symmetric and traceless tensors are called irreducible [1]. From Eqs. (6)-(9) it can be concluded that the reference tensors $\left.\mathbb{T}_{\left\langle\alpha_{i}\right\rangle}^{\prime}\right\rangle$ reflect the crystal symmetry

$f(\boldsymbol{Q})=f\left(\boldsymbol{Q} \boldsymbol{H}^{\mathrm{C}}\right) \Rightarrow \mathbb{T}_{\left\langle\alpha_{i}\right\rangle}^{\prime}=\boldsymbol{H}^{\mathrm{C}} * \mathbb{T}_{\left\langle\alpha_{i}\right\rangle}^{\prime} \quad \forall \boldsymbol{H}^{\mathrm{C}} \in S^{\mathrm{C}}$,

whereas the tensorial Fourier coefficients $\mathbb{V}_{\left\langle\alpha_{i}\right\rangle}^{\prime}$ have the sample symmetry

$f(\boldsymbol{Q})=f\left(\boldsymbol{H}^{\mathrm{S}} \boldsymbol{Q}\right) \Rightarrow \mathbb{V}_{\left\langle\alpha_{i}\right\rangle}^{\prime}=\boldsymbol{H}^{\mathrm{S}} * \mathbb{V}_{\left\langle\alpha_{i}\right\rangle}^{\prime} \quad \forall \boldsymbol{H}^{\mathrm{S}} \in S^{\mathrm{S}}$.

\subsection{Calculation of the Fourier coefficients}

In the context of a tensorial Fourier expansion of the codf the orthogonality relations are given by

$\int_{S O(3)} \mathbb{F}_{\left\langle\alpha_{i}\right\rangle}^{\prime}(\boldsymbol{Q}) \otimes \mathbb{F}_{\left\langle\alpha_{j}\right\rangle}^{\prime}(\boldsymbol{Q}) \mathrm{d} Q=\mathbb{O} \quad \forall i \neq j$

and

$\int_{S O(3)} \mathbb{F}_{\left\langle\alpha_{i}\right\rangle}^{\prime}(\boldsymbol{Q}) \otimes \mathbb{F}_{\left\langle\alpha_{i}\right\rangle}^{\prime}(\boldsymbol{Q}) \mathrm{d} Q=\frac{1}{2 \alpha_{i}+1} \boldsymbol{\Delta}_{\left\langle 2 \alpha_{i}\right\rangle}$.

$\boldsymbol{\Delta}_{\left\langle 2 \alpha_{i}\right\rangle}$ denotes the identity on irreducible tensors of rank $\alpha_{i}$.

A combination of Eqs. (8) and (16) gives the following set of linear equations

$\mathbb{J}_{\left\langle\alpha_{i}\right\rangle}^{\prime}=\mathbb{E}_{\left\langle 2 \alpha_{i}\right\rangle}\left[\mathbb{V}_{\left\langle\alpha_{i}\right\rangle}^{\prime}\right]$,

where

$\mathbb{J}_{\left\langle\alpha_{i}\right\rangle}^{\prime}=\int_{S O(3)} f(\boldsymbol{Q}) \mathbb{F}_{\left\langle\alpha_{i}\right\rangle}^{\prime}(\boldsymbol{Q}) \mathrm{d} Q$

and

$\mathbb{E}_{\left\langle 2 \alpha_{i}\right\rangle}=\int_{S O(3)} \mathbb{F}_{\left\langle\alpha_{i}\right\rangle}^{\prime}(\boldsymbol{Q}) \otimes \mathbb{F}_{\left\langle\alpha_{i}\right\rangle}^{\prime}(\boldsymbol{Q}) \mathrm{d} Q$.

Eq. (17) can now be used to simplify (20) and to solve the system of Eq. (18). Finally one obtains

$\mathbb{V}_{\left\langle\alpha_{i}\right\rangle}^{\prime}=\left(2 \alpha_{i}+1\right) \int_{S O(3)} f(\boldsymbol{Q}) \mathbb{F}_{\left\langle\alpha_{i}\right\rangle}^{\prime}(\boldsymbol{Q}) \mathrm{d} Q$.

For a set of discrete crystal orientations and corresponding volume fractions $\left\{\boldsymbol{Q}_{\beta}, v_{\beta}\right\}(\beta=1, \ldots, N)$ the codf can be approximated by

$f \approx \sum_{\beta=1}^{N} v_{\beta} \delta_{\boldsymbol{Q}_{\beta}}$.

Eqs. (21) and (22) imply the following formula for the texture coefficients

$\mathbb{V}_{\left\langle\alpha_{i}\right\rangle}^{\prime}=\left(2 \alpha_{i}+1\right) \sum_{\beta=1}^{N} v_{\beta} \boldsymbol{Q}_{\beta} * \mathbb{T}_{\left\langle\alpha_{i}\right\rangle}^{\prime}$.

If the orientation distribution function is uniform, then all tensorial Fourier coefficients vanish and $f(\boldsymbol{Q})=1$ holds.
Eqs. (11) and (23) show that for a single crystal orientation the norm of the coefficients is

$\left\|\mathbb{V}_{\left\langle\alpha_{i}\right\rangle}^{\prime}\right\|=2 \alpha_{i}+1$.

Based on the triangle inequality it can be concluded that for a textured polycrystal

$$
\begin{aligned}
\left\|\mathbb{V}_{\left\langle\alpha_{i}\right\rangle}^{\prime}\right\| & \leqslant\left(2 \alpha_{i}+1\right) \sum_{\beta=1}^{N} v_{\beta}\left\|\boldsymbol{Q}_{\beta} * \mathbb{T}_{\left\langle\alpha_{i}\right\rangle}^{\prime}\right\| \\
& =\left(2 \alpha_{i}+1\right) \sum_{\beta=1}^{N} v_{\beta}=\left(2 \alpha_{i}+1\right) .
\end{aligned}
$$

Hence the magnitude of the coefficients is generally bounded by

$\left\|\mathbb{V}_{\left\langle\alpha_{i}\right\rangle}^{\prime}\right\| \in\left[0,2 \alpha_{i}+1\right]$.

The values $\left\|\mathbb{V}_{\left\langle\alpha_{i}\right\rangle}^{\prime}\right\|$ are measures for the anisotropy degree of a polycrystal.

\subsection{Dimension and parameterization of irreducible tensors}

In the three-dimensional space a tensor of rank $R$ has $3^{R}$ independent components. If a tensor $(R \geqslant 2)$ is symmetric with respect to all pairs of indices, then the number of independent components reduces to

$\sum_{k=1}^{R+1} k=\frac{1}{2}(R+1)(R+2)$.

If a completely symmetric tensor is required to be traceless then the number of constraints is equal to the number of independent components of a symmetric tensor of rank $R-2$. As a result such an irreducible tensor has the dimension

$\sum_{k=1}^{R+1} k-\sum_{k=1}^{R-1} k=2 R+1$.

Hence, the number of independent components increases linearly with the tensor rank. In Table 1 the dimension of higher order tensors subjected to the aforementioned constraints is shown for different values of $R$.

As an example, nine independent components of a fourth-order irreducible tensor $\mathbb{V}_{\langle 4\rangle}^{\prime}$ are given

$$
\begin{array}{lll}
V_{1}^{4}=V_{1111}^{\prime}, & V_{2}^{4}=V_{1112}^{\prime}, & V_{3}^{4}=V_{1113}^{\prime}, \\
V_{4}^{4}=V_{1122}^{\prime}, & V_{5}^{4}=V_{1123}^{\prime}, & V_{6}^{4}=V_{1222}^{\prime}, \\
V_{7}^{4}=V_{1223}^{\prime}, & V_{8}^{4}=V_{2222}^{\prime}, & V_{9}^{4}=V_{2223}^{\prime} .
\end{array}
$$

The condition that $\mathbb{V}_{\langle 4\rangle}^{\prime}$ is traceless implies

$$
\begin{aligned}
& V_{1133}^{\prime}=-V_{1111}^{\prime}-V_{1122}^{\prime}=-V_{1}^{4}-V_{4}^{4}, \\
& V_{1233}^{\prime}=-V_{1112}^{\prime}-V_{1222}^{\prime}=-V_{2}^{4}-V_{6}^{4}, \\
& V_{1333}^{\prime}=-V_{1113}^{\prime}-V_{1322}^{\prime}=-V_{3}^{4}-V_{7}^{4}, \\
& V_{2233}^{\prime}=-V_{1122}^{\prime}-V_{2222}^{\prime}=-V_{4}^{4}-V_{8}^{4}, \\
& V_{2333}^{\prime}=-V_{1123}^{\prime}-V_{2223}^{\prime}=-V_{5}^{4}-V_{9}^{4}, \\
& V_{3333}^{\prime}=-V_{1133}^{\prime}-V_{2233}^{\prime}=V_{1}^{4}+2 V_{4}^{4}+V_{8}^{4} .
\end{aligned}
$$


Table 1

Number of independent components of $R$ th-order tensors due to different types of constraints

\begin{tabular}{lcccc}
\hline Rank $R$ & $\mathbb{V}$ General & $\mathbb{V}$ Traceless & $\mathbb{V}$ Symmetric & $\mathbb{V}$ Irreducible \\
\cline { 2 - 5 } & $\operatorname{dim} \mathbb{V}=3^{R}$ & $\operatorname{dim} \mathbb{V}=8 \times 3^{R-2}$ & $\operatorname{dim} \mathbb{V}=\frac{1}{2}(R+1)(R+2)$ & $\operatorname{dim} \mathbb{V}=2 R+1$ \\
\hline 2 & 9 & 8 & 6 & 5 \\
4 & 81 & 72 & 15 & 9 \\
6 & 729 & 648 & 28 & 13 \\
8 & 6561 & 5832 & 65 & 17 \\
10 & 59049 & 52488 & 66 & 21 \\
\hline
\end{tabular}

All other components of $\mathbb{V}_{\langle 4\rangle}^{\prime}$ can be derived by the condition that $\mathbb{V}_{\langle 4\rangle}^{\prime}$ is completely symmetric. Based on the aforementioned results, the scalar product of two fourth-order irreducible tensors $\mathbb{V}_{\langle 4\rangle}^{\prime}$ and $\mathbb{F}_{\langle 4\rangle}^{\prime}$ can be expressed by the corresponding components $V_{1 \ldots 9}^{4}$ and $F_{1 \ldots 9}^{4}$

$$
\begin{aligned}
\mathbb{V}_{\langle 4\rangle}^{\prime} \cdot \mathbb{F}_{\langle 4\rangle}^{\prime}= & F_{8}^{4} V_{1}^{4}+16 F_{2}^{4} V_{2}^{4}+12 F_{6}^{4} V_{2}^{4}+8 F_{3}^{4} V_{3}^{4}+4 F_{7}^{4} V_{3}^{4} \\
& +8 F_{8}^{4} V_{4}^{4}+16 F_{5}^{4} V_{5}^{4}+4 F_{9}^{4} V_{5}^{4}+12 F_{2}^{4} V_{6}^{4} \\
& +16 F_{6}^{4} V_{6}^{4}+4 F_{3}^{4} V_{7}^{4}+16 F_{7}^{4} V_{7}^{4}+8 F_{8}^{4} V_{8}^{4} \\
& +F_{1}^{4}\left(8 V_{1}^{4}+8 V_{4}^{4}+V_{8}^{4}\right)+F_{4}^{4}\left(8 V_{1}^{4}+22 V_{4}^{4}+8 V_{8}^{4}\right) \\
& +4 F_{5}^{4} V_{9}^{4}+8 F_{9}^{4} V_{9}^{4} .
\end{aligned}
$$

A similar formula holds for higher-order tensors.

\subsection{Irreducible tensors with a specific symmetry}

The reference tensors $\mathbb{T}_{\left\langle\alpha_{i}\right\rangle}^{\prime}$ have the crystal symmetry (see (14)), i.e.,

$\mathbb{T}_{\left\langle\alpha_{i}\right\rangle}^{\prime}=\boldsymbol{H}^{\mathrm{C}} * \mathbb{T}_{\left\langle\alpha_{i}\right\rangle}^{\prime} \quad \forall \boldsymbol{H}^{\mathrm{C}} \in S^{\mathrm{C}}$.

Reference tensors satisfying (29) can be constructed in the following way. Let $S^{\mathrm{C}}$ be given by the proper orthogonal tensors $\left\{\boldsymbol{H}_{\beta}^{\mathrm{C}}\right\}$ with $\beta=1, \ldots, N_{\mathrm{C}}$ and let $\mathbb{A}_{\left\langle\alpha_{i}\right\rangle}^{\prime}$ be an arbitrary irreducible tensor of rank $\alpha$. Then it can be shown that the tensor

$\mathbb{T}_{\left\langle\alpha_{i}\right\rangle}^{\prime}=\frac{1}{N_{\mathrm{C}}} \sum_{\beta=1}^{N_{\mathrm{C}}} \boldsymbol{H}_{\beta}^{\mathrm{C}} * \mathbb{A}_{\left\langle\alpha_{i}\right\rangle}^{\prime}$

fulfills Eq. (29).

In the present paper, a cubic crystal symmetry is considered. In this case the symmetry group $S^{\mathrm{C}}$ is given by 24 orthogonal tensors mapping the unit cube onto itself. For the cubic case the independent components have been determined by Guidi et al. [10]. For example, independent components of $\mathbb{T}_{\langle 4\rangle}$ are given by

$$
\begin{aligned}
& T_{1}^{4}=T_{1111}^{\prime}=2 a, \quad T_{2}^{4}=T_{1112}^{\prime}=0, \quad T_{3}^{4}=T_{1113}^{\prime}=0, \\
& T_{4}^{4}=T_{1122}^{\prime}=-a, \quad T_{5}^{4}=T_{1123}^{\prime}=0, \quad T_{6}^{4}=T_{1222}^{\prime}=0, \\
& T_{7}^{4}=T_{1223}^{\prime}=0, \quad T_{8}^{4}=T_{2222}^{\prime}=2 a, \quad T_{9}^{4}=T_{2223}^{\prime}=0 .
\end{aligned}
$$

The constant $a$ can be calculated by the normalization condition $\left\|\mathbb{T}_{\langle 4\rangle}\right\|=1: a=1 / \sqrt{30}$. The independent components of $\mathbb{T}_{\langle 6\rangle}$ are
$T_{1}^{6}=T_{111111}^{\prime}=2 a, \quad T_{2}^{6}=T_{11112}^{\prime}=0, \quad T_{3}^{6}=T_{11113}^{\prime}=0$,

$T_{4}^{6}=T_{111122}^{\prime}=-a, \quad T_{5}^{6}=T_{111123}^{\prime}=0, \quad T_{6}^{6}=T_{111222}^{\prime}=0$,

$T_{7}^{6}=T_{111223}^{\prime}=0, \quad T_{8}^{6}=T_{112222}^{\prime}=-a, \quad T_{9}^{6}=T_{112223}^{\prime}=0$,

$T_{10}^{6}=T_{122222}^{\prime}=0, \quad T_{11}^{6}=T_{122223}^{\prime}=0, \quad T_{12}^{6}=T_{222222}^{\prime}=2 a$,

$T_{13}^{6}=T_{22223}^{\prime}=0$.

The normalization condition $\left\|\mathbb{T}_{\langle 6\rangle}\right\|=1$ implies: $a=$ $1 / \sqrt{462}$.

\section{The evolution equation of the tensorial texture coefficients}

\subsection{Time derivative of orientational averages}

The Lagrangian form of the conservation law of the codf for regular points is given by

$f(\boldsymbol{Q}, t)=\operatorname{det}\left(\boldsymbol{G}\left(\boldsymbol{Q}_{0}, t\right)\right)^{-1} f_{0}\left(\boldsymbol{Q}_{0}\right)$

$[16,17]$, where $f_{0}\left(\boldsymbol{Q}_{0}\right)$ is the initial codf. $\boldsymbol{Q}_{0}$ denotes the initial orientation of a crystal having the orientation $\boldsymbol{Q}$ at time $t$. The dependence of $\boldsymbol{Q}$ on $\boldsymbol{Q}_{0}$ is assumed to be given by a one-to-one mapping $\boldsymbol{Q}=\boldsymbol{q}\left(\boldsymbol{Q}_{0}, t\right)$ with continuous $\boldsymbol{G}=$ $\partial \boldsymbol{q}\left(\boldsymbol{Q}_{0}, t\right) / \partial \boldsymbol{Q}_{0}$ and $\operatorname{det}(\boldsymbol{G})>0$.

For time dependent orientation distributions an orientational average of a quantity $\psi(\boldsymbol{Q})$ is given by

$\bar{\psi}(t)=\int_{S O(3)} f(\boldsymbol{Q}, t) \psi(\boldsymbol{Q}) \mathrm{d} Q$

and the corresponding time derivative is

$\dot{\bar{\psi}}(t)=\frac{\mathrm{d}}{\mathrm{d} t} \int_{S O(3)} f(\boldsymbol{Q}, t) \psi(\boldsymbol{Q}) \mathrm{d} Q$.

After substituting (31) into Eq. (33) and taking into account that the volume elements of the current and the initial codf are related by $\mathrm{d} Q=\operatorname{det}(\boldsymbol{G}) \mathrm{d} Q_{0}$ one obtains

$\dot{\bar{\psi}}(t)=\int_{S O(3)} f_{0}\left(\boldsymbol{Q}_{0}\right) \dot{\psi}\left(\boldsymbol{q}\left(\boldsymbol{Q}_{0}, t\right)\right) \mathrm{d} Q_{0}$.

Using again Eq. (31) and applying the chain rule finally gives

$\dot{\bar{\psi}}(t)=\int_{S O(3)} f(\boldsymbol{Q}, t) \frac{\partial \psi(\boldsymbol{Q})}{\partial \boldsymbol{Q}} \cdot \dot{\boldsymbol{Q}} \mathrm{d} Q$.

The reorientation velocity $\dot{\boldsymbol{Q}}$ is a constitutive quantity. It can be concluded that the local formulation of the balance equation of the codf implies that the time derivative of an 
orientational average is equal to the orientational average of the material time derivative.

\subsection{Evolution equation of the tensorial texture coefficients}

The evolution equation of the tensorial texture coefficients $\mathbb{V}_{\left\langle\alpha_{i}\right\rangle}^{\prime}$ (see (21)) can be derived based on Eq. (35). Eq. (35) implies that

$\dot{\mathbb{V}}_{\left\langle\alpha_{i}\right\rangle}^{\prime}(t)=\left(2 \alpha_{i}+1\right) \int_{S O(3)} f(\boldsymbol{Q}, t) \dot{\mathbb{F}}_{\left\langle\alpha_{i}\right\rangle}^{\prime}(\boldsymbol{Q}) \mathrm{d} Q$

holds. The time derivative of the Rayleigh product $\mathbb{F}^{\prime}(\boldsymbol{Q})=\boldsymbol{Q} * \mathbb{T}^{\prime}$ can be formalized by

$\dot{\mathbb{F}}_{\left\langle\alpha_{i}\right\rangle}^{\prime}(\boldsymbol{Q})=\dot{\boldsymbol{Q}} \boldsymbol{Q}^{-1} \otimes \mathbb{F}_{\left\langle\alpha_{i}\right\rangle}^{\prime}(\boldsymbol{Q})=\boldsymbol{\Omega} \otimes \mathbb{F}_{\left\langle\alpha_{i}\right\rangle}^{\prime}(\boldsymbol{Q})$,

where

$(\mathbf{\Omega} \otimes \mathbb{F})_{i j \ldots k}=\Omega_{i m} F_{m j \ldots k}+\Omega_{i m} F_{j m \ldots k}+\cdots+\Omega_{i m} F_{j k \ldots m}$.

In case of viscoplastic single crystals (without hardening) the skew tensor $\boldsymbol{\Omega}$ depends on the current crystal orientation and the velocity gradient $\boldsymbol{L}=\partial \boldsymbol{v} / \partial \boldsymbol{x}$. From Eqs. (36) and (37) it follows that the evolution equation of the texture coefficients is given by

$\dot{\mathbb{V}}_{\left\langle\alpha_{i}\right\rangle}^{\prime}(t)=\left(2 \alpha_{i}+1\right) \int_{S O(3)} f(\boldsymbol{Q}, t) \boldsymbol{\Omega}(\boldsymbol{L}(t), \boldsymbol{Q}) \otimes \mathbb{F}_{\left\langle\alpha_{i}\right\rangle}^{\prime}(\boldsymbol{Q}) \mathrm{d} Q$.

As an example $\dot{\mathbb{F}}_{\langle 4\rangle}^{\prime}(\boldsymbol{Q})=\boldsymbol{\Omega} \otimes \mathbb{F}_{\langle 4\rangle}^{\prime}(\boldsymbol{Q})$ is given here explicitly in terms of the axial vector $\boldsymbol{w}=\boldsymbol{\varepsilon}[\mathbf{\Omega}] / 2$ of $\boldsymbol{\Omega}$ where $\boldsymbol{\varepsilon}$ denotes the permutation tensor

$\dot{F}_{1}^{4}=+4 w_{1} F_{2}^{4}+4 w_{2} F_{3}^{4}$,

$\dot{F}_{2}^{4}=-w_{1} F_{1}^{4}+3 w_{1} F_{4}^{4}+3 w_{2} F_{5}^{4}+w_{3} F_{3}^{4}$,

$\dot{F}_{3}^{4}=-4 w_{2} F_{1}^{4}+3 w_{1} F_{5}^{4}-w_{3} F_{2}^{4}-3 w_{2} F_{4}^{4}$,

$\dot{F}_{4}^{4}=-2 w_{1} F_{2}^{4}+2 w_{1} F_{6}^{4}+2 w_{2} F_{7}^{4}+2 w_{3} F_{5}^{4}$,

$\dot{F}_{5}^{4}=-w_{1} F_{3}^{4}-3 w_{2} F_{2}^{4}+2 w_{1} F_{7}^{4}-2 w_{3} F_{4}^{4}-2 w_{2} F_{6}^{4}-w_{3} F_{1}^{4}$,

$\dot{F}_{6}^{4}=-3 w_{1} F_{4}^{4}+w_{1} F_{8}^{4}+w_{2} F_{9}^{4}+3 w_{3} F_{7}^{4}$,

$\dot{F}_{7}^{4}=-2 w_{1} F_{5}^{4}-2 w_{2} F_{4}^{4}+w_{1} F_{9}^{4}-3 w_{3} F_{6}^{4}-w_{2} F_{8}^{4}-2 w_{3} F_{2}^{4}$,

$\dot{F}_{8}^{4}=-4 w_{1} F_{6}^{4}+4 w_{3} F_{9}^{4}$,

$\dot{F}_{9}^{4}=-3 w_{1} F_{7}^{4}-w_{2} F_{6}^{4}-4 w_{3} F_{8}^{4}-3 w_{3} F_{4}^{4}$.

\subsection{The closure problem}

For numerical computations only a finite number of coefficients $\dot{\mathbb{V}}_{\left\langle\alpha_{i}\right\rangle}^{\prime}$ can be taken into account. Hence, for the solution of the evolution Eq. (39) it is necessary to estimate the distribution function $f(\boldsymbol{Q}, t)$ based on a limited number of texture coefficients. In the following this estimate is denoted by $\bar{f}(\boldsymbol{Q}, t)$. The estimate has to be consistent in the sense that $(5)_{1}$ and $(5)_{2}$ are fulfilled. It should be noted that a truncation of the series (8) would generally give an inconsistent estimate of the codf, because the constraint $(5)_{1}$ can be violated
$f(\boldsymbol{Q})=1+\sum_{i=1}^{L} \mathbb{V}_{\left\langle\alpha_{i}\right\rangle}^{\prime} \cdot \mathbb{F}_{\left\langle\alpha_{i}\right\rangle}^{\prime}(\boldsymbol{Q}) \ngtr 0$

if $L$ is finite. In the context of the representation of the codf by generalized harmonics this problem has been considered by Van Houtte [23].

The problem of estimating a distribution function based on incomplete data has no unique solution and therefore it is ill-posed. One way to single out a solution of the aforementioned problem is to maximize the informationtheoretic entropy of the estimate $\bar{f}(\boldsymbol{Q})$ of $f(\boldsymbol{Q})$ under the constraints that $\bar{f}(\boldsymbol{Q}, t)$ is normalized and that $\bar{f}(\boldsymbol{Q}, t)$ has the same leading texture coefficients as the function $f(\boldsymbol{Q}, t)$. The maximum entropy method or maximum entropy principle was introduced by Jaynes $[12,13]$ into the field of statistical mechanics.

The entropy of the orientation distribution function is defined by

$E=-\int_{S O(3)} f(\boldsymbol{Q}) \ln (f(\boldsymbol{Q})) \mathrm{d} Q \in(-\infty, 0]$.

A discussion of the general features of the texture entropy can be found in Schaeben [21]. For an application of the maximum entropy principle in the context of tensorial texture coefficients the reader is referred to Böhlke [4]. There it is shown that the estimate $\bar{f}(\boldsymbol{Q})$ has the following form

$\bar{f}(\boldsymbol{Q})=\exp \left(-\bar{V}_{0}-\sum_{i=1}^{L}\left(2 \alpha_{i}+1\right) \overline{\mathbb{V}}_{\left\langle\alpha_{i}\right\rangle}^{\prime} \cdot \mathbb{F}_{\left\langle\alpha_{i}\right\rangle}^{\prime}(\boldsymbol{Q})\right)$.

The multipliers $\bar{V}_{0}$ and $\overline{\mathbb{V}}_{\left\langle\alpha_{i}\right\rangle}^{\prime}$ have to be computed from the set of $1+\sum_{i=1}^{L}\left(2 \alpha_{i}+1\right)$ nonlinear equations

$1=\int_{S O(3)} \bar{f}(\boldsymbol{Q}) \mathrm{d} Q$

and

$\mathbb{V}_{\left\langle\alpha_{i}\right\rangle}^{\prime}=\left(2 \alpha_{i}+1\right) \int_{S O(3)} \bar{f}(\boldsymbol{Q}) \mathbb{F}_{\left\langle\alpha_{i}\right\rangle}^{\prime}(\boldsymbol{Q}) \mathrm{d} Q$

$(i=1, \ldots, L)$. For example, if $\mathbb{V}_{\langle 4\rangle}^{\prime}$ and $\mathbb{V}_{\langle 6\rangle}^{\prime}$ are known, then $L=2$ and the codf is estimated based on 22 texture coefficients, i.e., the 9 independent components of $\mathbb{V}_{\langle 4\rangle}^{\prime}$ and the 13 independent components of $\mathbb{V}_{\langle 6\rangle}^{\prime}$. In this case, the estimate (42) depends on the 23 parameters $\left\{\bar{V}_{0}, \mathbb{V}_{\langle 4\rangle}^{\prime}, \mathbb{V}_{\langle 6\rangle}^{\prime}\right\}$ which are determined by the 23 constraints (43), (44). In Section 4 the following estimates will be used in order to solve the differential equation of the texture coefficients

$$
\begin{aligned}
A \quad \bar{f}(\boldsymbol{Q}, t) & =\exp \left(-\bar{V}_{0}-9 \overline{\mathbb{V}}_{\langle 4\rangle}^{\prime}(t) \cdot \mathbb{F}_{\langle 4\rangle}^{\prime}(\boldsymbol{Q})\right), \\
B \quad \bar{f}(\boldsymbol{Q}, t) & =\exp \left(-\bar{V}_{0}-9 \overline{\mathbb{V}}_{\langle 4\rangle}^{\prime}(t) \cdot \mathbb{F}_{\langle 4\rangle}^{\prime}(\boldsymbol{Q})\right. \\
& \left.-13 \overline{\mathbb{V}}_{\langle 6\rangle}^{\prime}(t) \cdot \mathbb{F}^{\prime}(\boldsymbol{Q})\right), \\
C \quad \bar{f}(\boldsymbol{Q}, t) & =\exp \left(-\bar{V}_{0}-9 \overline{\mathbb{V}}_{\langle 4\rangle}^{\prime}(t) \cdot \mathbb{F}_{\langle 4\rangle}^{\prime}(\boldsymbol{Q})-\cdots\right. \\
& \left.-17 \overline{\mathbb{V}}_{\langle 8\rangle}^{\prime}(t) \cdot \mathbb{F}^{\prime}(\boldsymbol{Q})\right) .
\end{aligned}
$$




\section{Numerical example}

\subsection{Flow rule and lattice spin}

Distortions of rigid-viscoplastic single crystals can be modeled by the following set of equations:

$$
\begin{aligned}
& \mathbf{0}=\boldsymbol{D}^{\prime}-\boldsymbol{Q} \operatorname{sym}\left(\tilde{\boldsymbol{K}}\left(\boldsymbol{Q}^{\top} \tau^{\prime} \boldsymbol{Q}, \tau_{\alpha}^{\mathrm{C}}\right)\right) \boldsymbol{Q}^{\top}, \\
& \dot{\boldsymbol{Q}} \boldsymbol{Q}^{-1}=\boldsymbol{W}-\boldsymbol{Q} \operatorname{skw}\left(\tilde{\boldsymbol{K}}\left(\boldsymbol{Q}^{\top} \tau^{\prime} \boldsymbol{Q}, \tau_{\alpha}^{\mathrm{C}}\right)\right) \boldsymbol{Q}^{\top}
\end{aligned}
$$

[11]. $\boldsymbol{D}$ and $\boldsymbol{W}$ are the symmetric and the skew part of the velocity gradient $\boldsymbol{L}$. $\tau$ denotes Kirchhoff stress tensor, which is related to the Cauchy stress tensor $\sigma$ by $\tau=J \sigma$, where $J=\varrho_{0} / \varrho$ is the determinant of the deformation gradient. The internal variables $\tau_{\alpha}^{\mathrm{C}}$ are the critical resolved shear stresses in the different slip systems. In the present work, fcc single crystals are considered. For this specific class of materials, it is a reasonable assumption that the slip systems harden in an isotropic manner, i.e., $\tau_{\alpha}^{\mathrm{C}}=\tau^{\mathrm{C}}[15]$. The function $\tilde{\boldsymbol{K}}$ is assumed to be given by

$$
\begin{aligned}
& \tilde{\boldsymbol{K}}\left(\boldsymbol{Q}^{\top} \boldsymbol{\tau}^{\prime} \boldsymbol{Q}, \tau^{\mathrm{C}}\right)=\sum_{\alpha}^{\mathrm{N}} \dot{\gamma}_{\alpha}\left(\tau_{\alpha}, \tau^{\mathrm{C}}\right) \tilde{\boldsymbol{M}}_{\alpha}, \\
& \dot{\gamma}_{\alpha}\left(\tau_{\alpha}, \tau^{\mathrm{C}}\right)=\dot{\gamma}_{0} \operatorname{sgn}\left(\tau_{\alpha}\right)\left|\frac{\tau_{\alpha}}{\tau^{\mathrm{C}}}\right|^{n}, \\
& \tau_{\alpha}=\left(\boldsymbol{Q}^{\top} \boldsymbol{\tau}^{\prime} \boldsymbol{Q}\right) \cdot \tilde{\boldsymbol{M}}_{\alpha}
\end{aligned}
$$

[11]. The material parameter $n$ quantifies the strain rate sensitivity of the material. It is generally temperature dependent and can be estimated by strain rate jump experiments. At room temperature $n$ is usually in the range of 50-250. In the limit $n \rightarrow \infty$ a rate-independent behavior is obtained. The Schmid or slip system tensors $\tilde{\boldsymbol{M}}_{\alpha}=\tilde{\boldsymbol{d}}_{\alpha} \otimes \tilde{\boldsymbol{n}}^{\alpha}$ are rankone tensors, which are defined in terms of the slip directions $\tilde{\boldsymbol{d}}_{\alpha}$ and the slip plane normals $\tilde{\boldsymbol{n}}^{\alpha}$. In the case of an fcc single crystal at room temperature, the octahedral slip systems $\{111\}\langle 110\rangle$ have to be taken into account $(N=12)$.

\subsection{Simple shear}

As an example a simple shear deformation is considered. Due to the application of the Taylor assumption, each crystal is subjected to the same deformation process. In the case of simple shear the velocity gradient has the representation $\boldsymbol{L}=L_{12} \boldsymbol{e}_{1} \otimes \boldsymbol{e}_{2}$, where $\boldsymbol{e}_{1}$ is the shear direction and $\boldsymbol{e}_{2}$ is the shear plane normal. $L_{12}$ is set to be equal to $0.001 \mathrm{~s}^{-1}$. The following material parameters are used: $\tau^{\mathrm{C}}=20 \mathrm{MPa}, \dot{\gamma}_{0}=$ $0.001 \mathrm{~s}^{-1}, n=50$.

In the first step the texture evolution is computed based on 1000 single crystal orientations. The discrete distribution of the crystal orientations (not of the Euler angles) is initially uniform. Since hardening is not considered here the microstructure is described by 3000 internal variables (Euler angles) the evolution of which is governed by Eq. (48). In the following this model is referred to as Taylor model 1 (T1).

In the second step the texture evolution is computed again based on the Taylor assumption but instead repre- senting the codf by a discrete set of crystals the differential Eq. (39) is solved. This model is referred to as Taylor model 2 (T2). The differential Eq. (39) is numerically integrated for the three closures A, B, and C (see (45)-(47)). The initial values of the three texture coefficients are computed from the discrete initial orientation distribution used for the texture simulation with model $\mathrm{T} 1$. In the cases $\mathrm{T} 2 \mathrm{~A}, \mathrm{~T} 2 \mathrm{~B}$, and T2C the crystallographic texture is represented by 9,22 , and 39 internal variables (texture coefficients), respectively. It is worth to mention that this number of internal variables corresponds to 3 , about 8 , and 13 single crystals.

The numerical solution of (43), (44) is obtained by a damped form of Newton's method. The Jacobian of the system of nonlinear equations has been numerically determined. The numerical integration over the elementary region of Euler angles (cubic crystal symmetry) is performed by the TOMS Algorithm 698 [2].

In Fig. 1 the norms of the coefficients $\overline{\mathbb{V}}_{\langle 4\rangle}^{\prime}, \overline{\mathbb{V}}_{\langle 6\rangle}^{\prime}$, and $\overline{\mathbb{V}}_{\langle 8\rangle}^{\prime}$ according to model $\mathrm{T} 1$ are shown as a function of the von Mises equivalent strain. Furthermore, the norm of $\overline{\mathbb{V}}_{\langle 4\rangle}^{\prime}$ is shown for model T2A. It can be seen that the magnitude of $\bar{\nabla}_{\langle 4\rangle}^{\prime}$ is described accurately for equivalent strains less than 1 . The saturation of the texture is not captured by model T2A.

If the deformation field and the hardening state are homogeneous in the aggregate, then the Taylor factor can be used to relate the critical resolved shear stress $\tau^{\mathrm{C}}$ on the microscale to the macroscopic flow stress $\bar{\sigma}$ by $\bar{\sigma}=$ $\bar{M} \tau^{\mathrm{C}}$. The Taylor factor can be estimated by $\bar{M} \approx \int_{S O(3)}$ $\sum_{\alpha}^{N}\left|\dot{\gamma}_{\alpha}\right| \mathrm{d} Q / \overline{\bar{\varepsilon}}$ where $\dot{\bar{\varepsilon}}$ denotes the equivalent strain rate on the macroscale. For a precise definition of the Taylor in the rate-dependent and the rate-independent case see [3]. In Fig. 2 the Taylor factor $\bar{M}$ is plotted for model T2A. The prediction of $\bar{M}$ is correct as far as the equivalent strain is less than 0.3 .

In Fig. 3 the norms of $\overline{\mathbb{V}}_{\langle 4\rangle}^{\prime}$ and $\overline{\mathbb{V}}_{\langle 6\rangle}^{\prime}$ are shown for the closure T2B. The corresponding predictions of model $\mathrm{T} 1$ are also shown. It can be seen that the prediction of the evolution concerning the coefficient $\overline{\mathbb{V}}_{\langle 4\rangle}^{\prime}$ by T2B

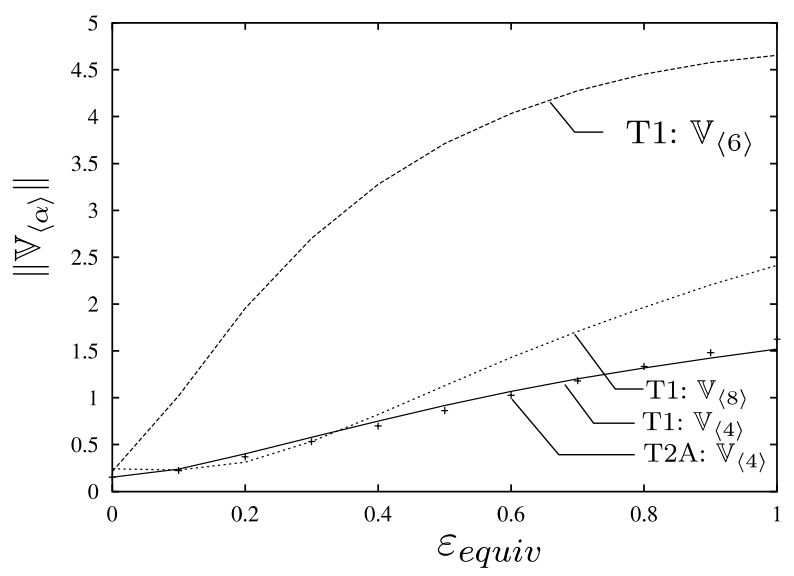

Fig. 1. Norm of the texture coefficients predicted by model T1 and model T2A. 


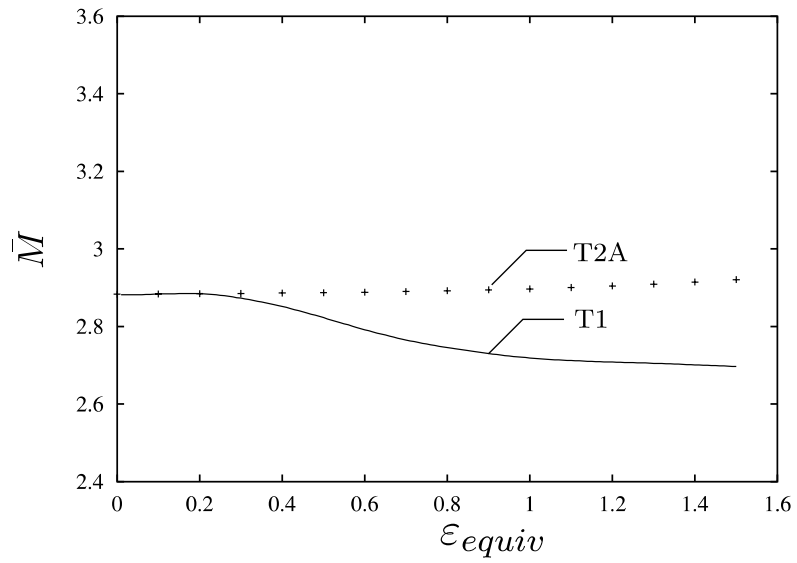

Fig. 2. Taylor factor predicted by model $\mathrm{T} 1$ and model T2A.

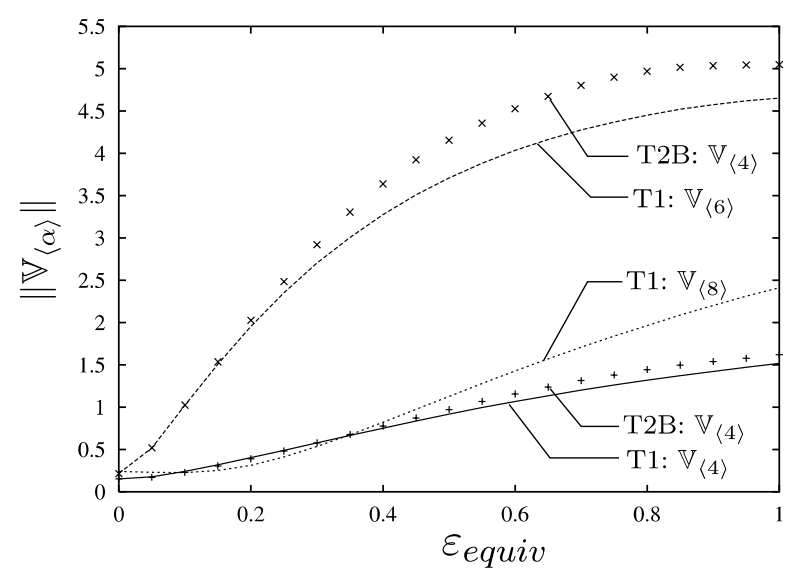

Fig. 3. Norm of the texture coefficients predicted by model T1 and model T2B.

corresponds to the predictions of model T1. For the coefficient $\overline{\mathbb{V}}_{\langle 6\rangle}^{\prime}$ model T2B predicts the initial slope of the curve correctly and describes also the saturation of the texture. The magnitude of the coefficients is overestimated for large strains. Fig. 4 indicates that model T2B describes the vari-

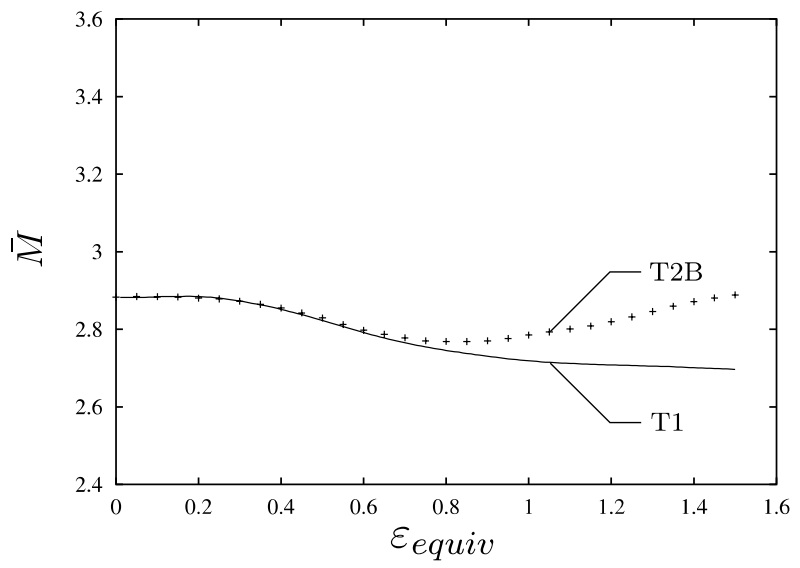

Fig. 4. Taylor factor predicted by model T1 and model T2B.

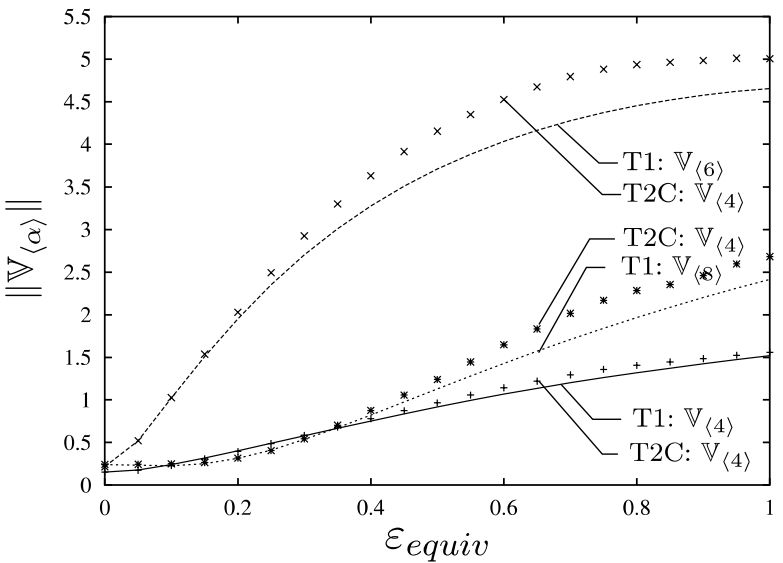

Fig. 5. Norm of the texture coefficients predicted by model T1 and model T2C.

ation of the Taylor factor over a larger range of deformations compared to model T2A. The prediction of $\bar{M}$ is correct as far as the equivalent strains less than 0.7.

Fig. 5 shows the norms of $\overline{\mathbb{V}}_{\langle 4\rangle}^{\prime}, \overline{\mathbb{V}}_{\langle 6\rangle}^{\prime}$, and $\overline{\mathbb{V}}_{\langle 6\rangle}^{\prime}$ predicted by model $\mathrm{T} 2 \mathrm{C}$ together with those of model $\mathrm{T} 1$. It can be seen that the prediction of the evolution concerning the coefficient $\overline{\mathbb{V}}_{\langle 4\rangle}^{\prime}$ by T2C again corresponds to the predictions of model T1. For the coefficients $\overline{\mathbb{V}}_{\langle 6\rangle}^{\prime}$ and $\overline{\mathbb{V}}_{\langle 8\rangle}^{\prime}$ model T2C predicts the initial slope of the curves correctly and describes also the saturation. The magnitude of the coefficients is overestimated for large strains. Fig. 6 indicates that model T2C describes the evolution of the Taylor factor as far as the equivalent strain is less than 0.9.

In Fig. 7 the codf predicted by model $\mathrm{T} 1$ is shown in the Euler angle space for an equivalent strain of 0.5. (Only the $90^{\circ}$ sections are shown for simplicity.) The codf has been estimated by means of the maximum entropy method based on the fourth, sixth, and eighth-order coefficients using the discrete orientations given by model $\mathrm{T} 1$. In Fig. 8 the corresponding plot is shown for model T2B. It can be seen that the main features of the texture evolution

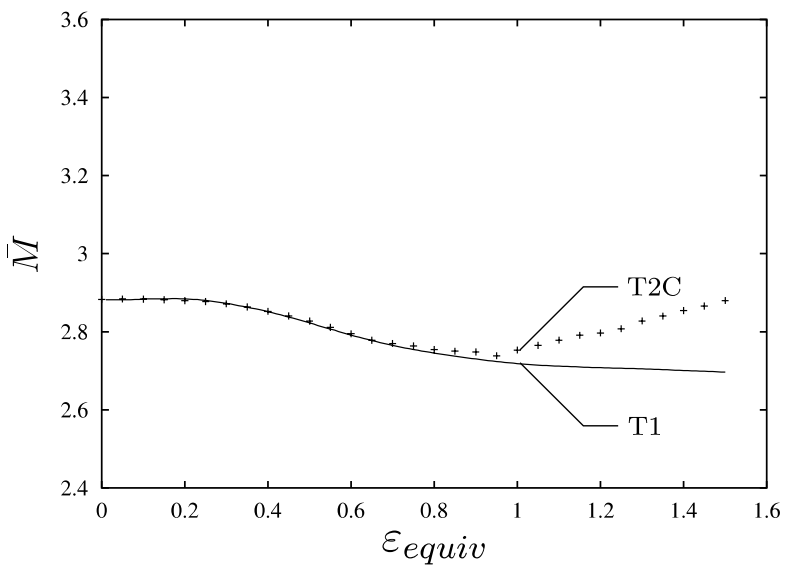

Fig. 6. Taylor factor predicted by model $\mathrm{T} 1$ and model T2C. 

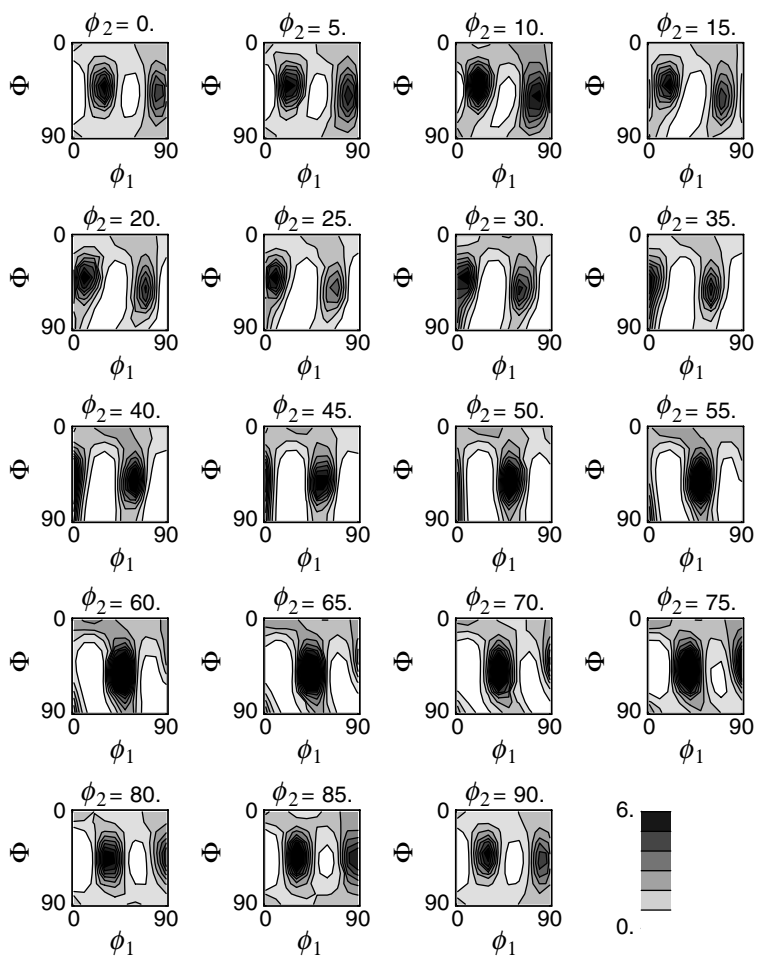

Fig. 7. The codf predicted by model T1 (simple shear, equivalent strain $0.5)$.
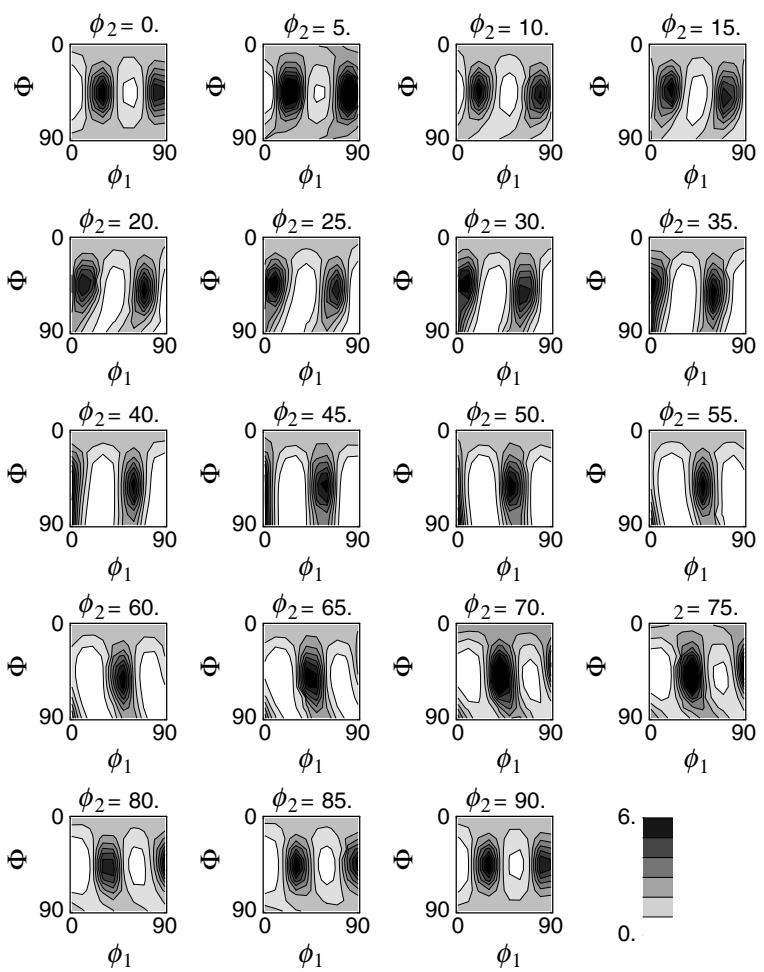

Fig. 8. The codf predicted by model T2B (simple shear, equivalent strain $0.5)$.

given by model T1 (3000 internal variables) are reproduced by model T2B (22 internal variables).

\section{Summary}

Based on the conservation law of the codf the differential equation of the tensorial texture coefficients has been derived and formulated in a coordinate-free setting. The evolution equation of each coefficient depends on the complete codf and the lattice spin, which is a constitutive quantity. For the solution of the differential equation based on a finite number of coefficients, the codf has to be estimated. The problem of estimating the codf based on a finite number of coefficients is generally ill-posed. One way to single out a solution is given by the application of the maximum entropy method [4]. In the present paper, this maximum entropy approach is used for the solution of the evolution equation governing the tensorial texture coefficients. Based on different closures with an increasing number of coefficients the differential equation is numerically solved for a simple shear deformation. It is shown that the evolution of the codf and of the Taylor factor is reproduced for moderate deformations if at least the 6th-order coefficients are taken into account. In contrast to formulations of the Taylor model, which are based on discrete orientation distributions, the approach suggested here allows to model the evolution of the codf by a much smaller number of internal variables.

\section{References}

[1] Adams B, Boehler J, Guidi M, Onat E. Group theory and representation of microstructure and mechanical behavior of polycrystals. J Mech Phys Solids 1992;40(4):723-37.

[2] Berntsen J, Espelid T. Algorithm 698: DCUHRE: An adaptive multidimensional integration routine for a vector of integrals. ACM Trans Math Softw 1991;17(4):452-6.

[3] Böhlke T. The Voigt bound of the stress potential of isotropic viscoplastic FCC polycrystals. Arch Mech 2004;56(6):423-43.

[4] Böhlke T. Application of the maximum entropy method in texture analysis. Comp Mat Sci 2004;32:276-83.

[5] Böhlke T, Bertram A. Crystallographic texture induced anisotropy in copper: An approach based on a tensorial Fourier expansion of the codf. J Phys IV 2003;105:167-74.

[6] Böhlke T, Bertram A, Krempl E. Modeling of deformation induced anisotropy in free-end torsion. Int J Plast 2003;19:1867-84.

[7] Bunge H-J. Zur Darstellung allgemeiner Texturen. Z Metallkde 1965;56:872-4.

[8] Bunge H-J. Texture analysis in material science. Göttingen: CuvillerVerlag; 1993.

[9] Gel'fand I, Minlos R, Shapiro Z. Representations of the rotation and Lorentz groups and their applications. Oxford: Pergamon Press; 1963.

[10] Guidi M, Adams B, Onat E. Tensorial representation of the orientation distribution function in cubic polycrystals. Textures Microstruct 1992;19:147-67.

[11] Hutchinson J. Bounds and self-consistent estimates for creep of polycrystalline materials. Proc Royal Soc London A 1976;348:101-27.

[12] Jaynes E. Information theory and statistical mechanics. Phys Rev 1957;106:620-30

[13] Jaynes E. Information theory and statistical mechanics II. Phys Rev 1957;108:171-90.

[14] Kalidindi S, Bronkhorst C, Anand L. Crystallographic texture evolution in bulk deformation processing of fcc metals. J Mech Phys Solids 1992;40(3):537-69. 
[15] Kocks U, Mecking H. Physics and phenomenology of strain hardening: The FCC case. Progr Mat Sci 2003;48:171-273.

[16] Kumar A, Dawson P. Simulation of texture evolution with finite elements over orientation space-I, Development. Comp Meth Appl Mech Eng 1996;130:227-46.

[17] Kumar A, Dawson P. Simulation of texture evolution with finite elements over orientation space-II, Application to planar crystals. Comp Meth Appl Mech Eng 1996;130:247-61.

[18] Mathur K, Dawson P. On modeling the development of crystallographic texture in bulk forming processes. Int J Plast 1989;5:67-94.

[19] Miehe C, Schröder J, Schotte J. Computational homogenization in finite plasticity, Simulation of texture development in polycrystalline materials. Comp Meth Appl Mech Eng 1999;171:387-418.

[20] Roe R. Description of crystalline orientation of polycrystalline materials. III. General solution to pole figure inversion. J Appl Phys 1965;36:2024-31.
[21] Schaeben H. Diskrete mathematische Methoden zur Berechnung und Interpretation von kristallographischen Orientierungsdichten. DGM Informationsgesellschaft mbH. Oberursel 1994.

[22] Reese S, Svendsen B. On the use of evolving structure tensors to model initial and induced elastic and inelastic anisotropy at finite deformation. J. Phys. IV France 2003;105:31-7.

[23] van Houtte P. The use of a quadratic form for the determination of nonnegative texture functions. Textures Microstruct 1983;6:1-20.

[24] Zheng Q-S, Fu Y-B. Orientation distribution functions for microstructures of heterogeneous materials: I Directional distribution functions and irreducible tensors. Appl Math Mech 2001;22(8): 865-84.

[25] Zheng Q-S, Fu Y-B. Orientation distribution functions for microstructures of heterogeneous materials: II Crystal distribution functions and irreducible tensors restricted by various material symmetries. Appl Math Mech 2001;22(8):885-903. 